\title{
Brain MR Diffusion Tensor Imaging for Multi-Site Studies: A Review
}

\author{
Youngseob Seo*1, 2 \\ ${ }^{1}$ Center for Medical Metrology, Korea Research Institute of Standards and Science, Korea
}

${ }^{2}$ Department of Medical Physics, University of Science and Technology, Korea

Received: January 12, 2018; Published: January 18, 2018

*Corresponding author: Youngseob Seo, Center for Medical Metrology, Korea Research Institute of Standards and Science, Daejeon, Department of Radiology, University of Science and Technology, Daejeon, 267 Gajeong-Ro, Yuseong-Gu, Daejeon 34113, Republic of Korea, Tel: 82-42-868-5479;

Fax: 82-42-868-5058; Email: Koreayseo@kriss.re.kr to yseo@kriss.re.kr

\begin{abstract}
This article provides a review of quality assurance and control issues in multi-site diffusion tensor imaging (DTI) studies for the evaluation of microstructural damage to white matter structures. In order to perform comparatively quantitative analysis of tensor metrics, tensor data must be of high quality and reproducible which requires documentation of scanner stability, minimization of artifacts, and optimization and standardization of image parameters. I review various practical aspects of DTI which impact quality of DTI with the focus on the quantification of tensor metrics.
\end{abstract}

Keywords: Diffusion tensor imaging; cerebral white matter; Multi-site

Abbreviations: DTI: Diffusion tensor imaging; FA: Fractional Anisotropy; SNR: Signal-Noise Ratio; MD: Mean Diffusivity

\section{Introduction}

Diffusion tensor imaging (DTI) [1] is sensitive to microstructural changes that occur in cerebral white matter with normal maturation and aging and in certain disease states [2-4]. Multi-site DTI studies decrease the length of time needed to accrue statistically sufficient numbers of patients with diverse conditions of varying severity. When comparing diffusion tensor data from multiple sites or even from different MR units at the same site, differences in tensor metrics may result from different hardware platforms, software releases, and imaging parameters. These techniquerelated effects may mask or mimic biological changes in white matter. Conducting a multi-site study using quantitative analysis of diffusion tensor data requires a uniform quality control procedure to ensure that data from different sites are not biased and can be combined for statistical analysis; and data of substandard quality are excluded. This review focuses on optimization of tensor data such that fractional anisotropy (FA) and diffusivity measurements from multiple sites and vendors can be compared. I identify various practical issues related to tensor data quality and compatibility, discuss how a quality control program may address these problems, and explore further research that may be needed in this area.

\section{Sampling Schemes}

The uncertainty of DTI metrics depends on the sampling scheme, the orientation and eigenvalues of the measured diffusion tensor, and signal-to-noise ratio (SNR). Images are usually collected with $\mathrm{b}=0 \mathrm{~s} / \mathrm{mm} 2$ and with high $\mathrm{b}$-values ( $\geq 600 \mathrm{~s} / \mathrm{mm} 2)$ in at least 6 directions. Computer simulations suggest that a minimum of 20 encoding directions are needed for robust FA quantification, and 30 directions are needed for reliable determination of the primary eigenvector [5]. Further increases in gradient directions do not improve reproducibility when using the single tensor model commonly used in clinical practice [4]. In a multi-vendor, multi-site study, it may not be practical to ensure that all sites use exactly the same sampling scheme. It may be more practical to minimize the difference in sampling scheme by using a sufficiently large number of sampling directions.

\section{Assessment of SNR}

As a rule, disorders of neurocognitive function and disease states that affect the white matter cause FA to decrease. Both the accuracy and precision of FA measurements are affected by SNR [68]. Regions with low FA are more prone to bias due to noise than regions with high FA. However, the mean diffusivity (MD) is less sensitive to noise. The variance of FA as a function of SNR for the widely used Jones30 gradient sampling scheme has previously been calculated for different FA values [9]. Minimum SNR requirements can be established for bias-free measurements in clinical practice. The SNR should be measured from anatomical structures of interest as SNR thresholds for FA bias differ with the inherent FA 
of the tissue being studied. The use of multiple $b=0$ images serves to decrease the level of noise in the tensor data set and at least one $\mathrm{b}=0$ acquisition for every 8 gradient directions are suggested [10]. In a multi-site study, it is important to verify that sites are following the specification on multiple $b=0$ acquisitions.

\section{Image Distortions and Motion Artifacts}

Eddy currents are a well-known problem in DTI which typically involves data readout using single-shot echo planar imaging (EPI) acquisitions. Gradient oscillations of the EPI technique induce eddy currents which can cause a delay of the echoes in the readout leading to image ghosting [11]. In routine preventative maintenance performed in the MR, checks of the eddy current compensation hardware and software will ensure the ghosting is kept to a minimum. The use of EPI readout in DTI makes the images sensitive to B0 inhomogeneity. Local B0 changes cause the location of structures to shift in the fold-over (PE) direction. Multi-site studies should use the same fat shift direction, along with the same parallel imaging acceleration factor and acquisition bandwidths. Motion-related signal losses include those caused by mechanical vibration of the scanner table, cardiac-induced pulsatility, and other bulk patient motion. The amplitude of the motion depends on the patient weight and may vary between scanners.

\section{Comparison of MR Scanners}

Acquisition of high-quality DTI data in reasonable times requires parallel imaging which is supported by all commercial MR vendors. The imaging reconstruction technique varies between vendors. While SENSE [12] used by Philips unfolds in image space, GRAPPA [13] used by Siemens uses a k-space synthesis technique based on SMASH [14] while ASSET used by GE employs the same principles as SENSE. Standardized imaging parameters can be used for multisite studies.

\section{Measurement Compatibility}

Direct comparison of FA measurements at $1.5 \mathrm{~T}$ and $3 \mathrm{~T}$ using identical pulse sequence parameters have yielded conflicting results [15-17]. In regions of high FA, FA is slightly higher at 3T than at $1.5 \mathrm{~T}$ while regions of the brain with intrinsically lower FA have higher FA at $1.5 \mathrm{~T}$ than $3 \mathrm{~T}$. SNR is lower at $1.5 \mathrm{~T}$ and low FA regions are more prone to bias due to noise than are regions of high FA. In studying subjects ranging in age from 1 to 24 years, our preliminary data suggests that FA values to be similar for $b=700$ and $1000 \mathrm{~s} /$ $\mathrm{mm} 2$, although axial and radial diffusivities are not. There is a fast decaying component at very low b-values which is related to perfusion and flow effects $[18,19]$. The non-monoexponential nature of diffusion signal decay also contributes to the dependence on the b-value of both FA and MD measurements.

\section{Quality Control (QC) Program Recommendations}

Qualitative assessment of image quality is not adequate when tensor data is being quantitatively assessed. There are no wellestablished or recommended QA or QC protocols for DTI, and QC procedures are not yet offered by the MR vendors. There is a need to develop QA procedures appropriate for DTI that can be used at individual sites and for multi-site studies which ensure that technical problems with the MR scanner are minimized. Uniform QA protocols assure that data from each participating sites are correctly calibrated and only data with sufficient quality are included. Inclusion of poor data will lead to negative findings or false findings resulting in Type 1 or 2 errors in group comparisons. FA and diffusivity from various manufacturers may vary substantially with minor differences in pulse sequence parameters [20]. For studies involving DWI and DTI, scanning parameters need to be standardized as closely as possible and scanners calibrated. There are several advantages of using the ACR phantom in a multisite setting for DTI QA. This phantom is widely available and used regardless of MR vendors and platforms for accreditation purposes.

\section{Conclusion}

Multi-site, multi-vendor studies using DTI may suffer from systematic differences in measurement results due to differences in pulse sequence implementation by different vendors. Individual scanners must meet high performance standards such as low eddy current distortions and sufficiently high SNR. Technical expertise is needed at each site to correctly implement the technique according to study specification and to quickly address scanning quality problems when they occur. At present, there are no formal recommendations for quality assurance or control procedure for multi-site DTI studies. One practical approach is to use an isotropic phantom with internal structures to check the scanner performance and to scan the same human phantom periodically to verify the reproducibility of the measurements. Individual data sets should be evaluated for SNR and artifacts before inclusion. Commitment and compliance to a quality assurance protocol containing these elements will ensure good scanning quality. High quality DTI studies for multi-site trials are within reach for investigators interested in applying this exciting technique.

\section{References}

1. Basser PJ, Mattiello J, LeBihan D (1994) MR diffusion tensor spectroscopy and imaging. Biophys J 66(1): 259-267.

2. Salat DH, Tuch DS, Hevelone ND, Fischl B, Corkin S, et al. (2005) Agerelated changes in prefrontal white matter measured by diffusion tensor imaging. Ann N Y Acad Sci 1064: 37-49.

3. Rollins NK (2007) Clinical applications of diffusion tensor imaging and tractography in children. Pediatr Radiol 37(8): 769-780.

4. Feldman HM, Yeatman JD, Lee ES, Barde LH, Gaman-Bean S (2010) Diffusion tensor imaging: a review for pediatric researchers and clinicians. J Dev Behav Pediatr 31(4): 346-356.

5. Jones DK (2004) the effect of gradient sampling schemes on measures derived from diffusion tensor MRI: a Monte Carlo study. Magn Reson Med 51(4): 807-815.

6. Pierpaoli C, Basser PJ (1996) toward a quantitative assessment of diffusion anisotropy. Magn Reson Med 36(6): 893-906.

7. Poonawalla AH, Zhou XJ (2004) Analytical error propagation in diffusion anisotropy calculations. J Magn Reson Imaging 19(4): 489-498.

8. Farrell JA, Landman BA, Jones CK, Smith SA, Prince JL, et al. (2007) Effects of signal-to-noise ratio on the accuracy and reproducibility of diffusion tensor imaging-derived fractional anisotropy, mean diffusivity, and principal eigenvector measurements at 1.5 T. J Magn Reson Imaging 26(3): 756-67. 
9. Chang LC, Koay CG, Pierpaoli C, Basser PJ (2007) Variance of estimated DTI-derived parameters via first-order perturbation methods. Magn Reson Med 57(1): 141-149.

10. Jones DK, Horsfield MA, Simmons A (1999) Optimal strategies for measuring diffusion in anisotropic systems by magnetic resonance imaging. Magn Reson Med 42(3): 515-525.

11. Le Bihan D, Poupon C, Amadon A, Lethimonnier F (2006) Artifacts and pitfalls in diffusion MRI. J Magn Reson Imaging 24(3): 478-488.

12. Pruessmann KP, Weiger M, Scheidegger MB, Boesiger P (1999) SENSE: sensitivity encoding for fast MRI. Magn Reson Med 42(5): 952-962.

13. Griswold MA, Jakob PM, Heidemann RM, Nittka M, Jellus V, et al. (2002) Generalized autocalibrating partially parallel acquisitions (GRAPPA) Magn Reson Med 47(6): 1202-1210.

14. Sodickson DK, Manning WJ (1997) Simultaneous acquisition of spatial harmonics (SMASH): fast imaging with radiofrequency coil arrays. Magn Reson Med 38(4): 591-603.

15. Hunsche S, Moseley ME, Stoeter P, Hedehus M (2001) Diffusion-tensor MR imaging at 1.5 and $3.0 \mathrm{~T}$ : initial observations. Radiology 221(2): 550556.

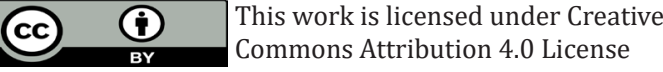

Submission Link: http://biomedres.us/submit-manuscript.php
16. Alexander AL, Lee JE, Wu YC, Field AS (2006) Comparison of diffusion tensor imaging measurements at $3.0 \mathrm{~T}$ versus $1.5 \mathrm{~T}$ with and without parallel imaging. Neuroimaging Clin N Am 16(2): 299-309.

17. Fushimi Y, Miki Y, Okada T, Yamamoto A, Mori N, et al. (2007) Fractional anisotropy and mean diffusivity: comparison between 3.0-T and 1.5$\mathrm{T}$ diffusion tensor imaging with parallel imaging using histogram and region of interest analysis. NMR Biomed 20(8): 743-748.

18. Le Bihan D, Breton E, Lallemand D, Grenier P, Cabanis E, et al. (1986) MR imaging of intravoxel incoherent motions: application to diffusion and perfusion in neurologic disorders. Radiology 161(2): 401-407.

19. Sasaki M, Yamada K, Watanabe Y, Matsui M, Ida M, et al. (2008) Variability in absolute apparent diffusion coefficient values across different platforms may be substantial: a multivendor, multi-institutional comparison study. Radiology 249(2): 624-630.

20. Chavez S, Storey P, Graham SJ (2009) Robust correction of spike noise: application to diffusion tensor imaging. Magn Reson Med 62(2): 510519.

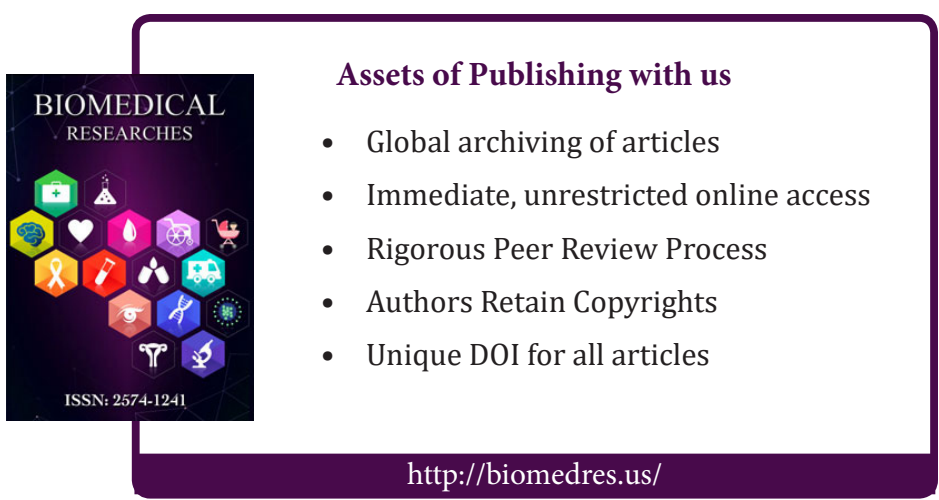

